\title{
A NOTE ON THE CLINICAL DIAGNOSIS OF RICKETS IN INFANCY.
}

\author{
BY \\ HELEN MACKAY, M.D., M.R.C.P.
}

The diagnosis of rickets may be based on any one of the following : (1) Examination of the blood, i.e., estimation of the blood phosphorus and blood calcium; (2) histological or (3) radiographic examination of the bones; (4) clinical examination of the patient.

It would seem probable that defective laying down of calcium in the growing bones results from an upset in the balance and the absolute amounts of the calcium and phosphorus salts in the blood, so that presumably the earliest diagnosis will in the future be based on blood examination and not on the secondary changes in the bones. Howland and Kramer claim that if the calcium concentration multiplied by the phosphorus concentration in milligrams per $100 \mathrm{cc}$. is less than 30 , there is always rickets present, that if this figure is between 30 and 40 the rickets is slight or healing. In the present stage of our knowledge, however, the generally accepted criterion of rickets is the histological one, i.e., the presence of excessive osteoid tissue, irregularity of the columns of cartilage cells, disorganisation at the epiphysial line and other pathological changes in the growing bones. As the bones, however, cannot be examined histologically during life, we are usually dependent on the evidence of changes revealed either by radiographic or by clinical examination-and these must of necessity be grosser and late: manifestations of the disease than those revealed by microscopic examination.

During the course of the investigation into infantile rickets carried out in Vienna under the auspices of the Medical Research Council and the Lister Institute of Preventive Medicine in the early post-war years, the writer undertook daily examinations of infants with the object of observing and recording the development of rachitic signs.

As regards the clinical diagnosis, it is now established that no reliance can be placed upon such general signs as pallor, sweating, flabbiness of muscles or large abdomen. All these may appear in many different conditions of ill-health, and moreover, the general health may be actually improving at the time that bone changes are becoming manifest. The diagnosis must, therefore, be based on evidence of bone changes alone, and, in the opinion of the writer, the most important of these for early diagnosis of infantile rickets are an enlarged rosary and craniotabes. Craniotabes is much more common in an infant developing rickets at four, five, or six months old than in one in whom the disease does not make its appearance until after say seven months, since the rate of growth of the skull diminishes rapidly. Craniotabes may be defined as softening of the cranial bones which develops after birth, and must always be distinguished from congenital softness of the bones-often a difficult matter. Two points of value in the differential diagnosis are that (1) craniotabes is usually asymmetrical, congenital softness symmetrical, (2) craniotabes usually first makes its appearance behind the ears about the anterior ends of the ocipito-parietal suture, congenital softness, if present at all, is usually well marked over the vertex, particularly about the posterior fontanelle. Craniotabes can be found in London infants with moderate frequency if it is systematically looked for, but its significance for 
the diagnosis of rickets is far more widely recognised on the Continent than in this country. (The grounds for considering craniotabes a rachitic manifestation are set out elsewhere. See Studies of Rickets in Vienna, 1919-1922. Medical Research Council Special Report Series, No. 77, p. 130.)

The presence of a pathologically enlarged rosary must be determined by palpation-no measurement can be given to divide the normal from the pathological, and each observer must learn for himself what are the limits of the normal. Moreover, an enlarged rosary like enlarged epiphyses is present both in rickets and scurvy.

During the Viennese investigation, the writer with Dr. E. Dalyell examined daily in the post mortem room the bodies of infants under 12 months of age, noting in each case the size of rosary, the presence or absence of craniotabes, size of epiphyses, etc. The diagnoses were then tested histologically. In 84 cases sections of the costo-chondral junctions were cut by Dr. Feller. Fourteen of the series showed a degree of bone change before autopsy held to justify a clinical diagnosis of rickets-of these 12 were histologically rachitic and two were cases of scurvy. Of the remaining 70 in the series, 13 were histologically rachitic though no definite signs of the condition were found in the bodies before autopsy. The system of notation for the rosary was $0, \frac{1}{2}, \frac{1}{2}-1,1,1-1 \frac{1}{2}, 1 \frac{1}{2}$, etc. It was found that many infants with a rosary of $1 \frac{1}{2}$ or more had other clinical evidence of rickets so that there was no doubt of the correctness of the diagnosis, and, therefore, only eight with beading of this grade were examined histologically. Of the eight, six were histologically rickets and the remaining two scurvy. Seven cases with craniotabes were examined and all had histoloyical evidence of rickets. By frequent examinations of living children it was established that, taking craniotabes and a rosary of grade $1 \frac{1}{2}$ upwards as evidence of rickets, the clinical diagnosis could frequently be made in infants before there were any radiographic signs of the disease. When it is remembered that rachitic changes tend to develop first at those sites where there is most rapid bone growth, and that in young infants the skull bones and the ribs are growing much more rapidly than the limb bones (i.e., those used for radiographic diagnosis for reasons of photographic technique), it is not surprising that an enlarged rosary or craniotabes can often be detected before there is evidence of rachitic change in the lower end of the radius and ulna.

As regards other clinical sign sof rickets in infants:-Bossing of the head, unless it is well marked, is difficult to diagnose with certainty on account of the wide normal variations in the shape of the head, and is, therefore, only occasionally helpful for early diagnosis.

Enlargement of the wrist is usually a somewhat later sign than enlargement of the costo-chondral junctions, and one must also bear in mind that there occurs normally between eight and 12 months of age an increase in prominence at the wrist which is sometimes mistaken for rachitic enlargement. By the time there is definite clinical enlargement of the wrist there is usually also radiographic evidence of the disease.

Bending of the limb bones is a relatively late sign and the degree of normal curvature of the tibia varies considerably so that a normal curve is frequently mistaken for a pathological one. A slight grade of thoracic deformity is often a fairly early sign, but this alone is insufficient for a diagnosis of rickets, since this deformity may arise in any young infant as a result, for example, of some respiratory embarrassment or persistent abdominal distension, though more likely to develop in a rachitic infant. 
In the course of the present investigation at the Queen's Hospital for Children, which is described in the previous paper, all three observers have based their clinical diagnosis on evidence of changes in the bones, but many cases have been passed by W.K. and H.S. as normal, which would have been classed by H.M. as rachitic taking so far as possible the standard test by histological investigtion in Vienna. Definite craniotabes or a rosary of $1 \frac{1}{2}$, with or without enlarged epiphyses, cranial bossing or thoracic deformity, was regarded as evidence of rickets.

There is no doubt, in the opinion of the writer, that the incidence of very slight grades of rickets is very much higher than the figures based on radiographic diagnosis would indicate. In the group of children examined by her at the Queen's Hospital for Children, 12 had clinical signs of rickets, though only five of them had definite radiographic evidence of the disease and in a sixth the evidence was doubtful. Every child in this group with radiographic signs of rickets had already been diagnosed clinically. The number showing histological changes indicative of the disease would undoubtedly be still larger.

Although clinical diagnosis can be made early it will always be subjective as it is not possible to convey in words the degree of beading or of epiphysial enlargement which is pathological : for example, prominence at the wrist which is within normal limits at one year would be pathological at five months, hence the very wide discrepancies in clinical diagnosis.

The only clinical sign which can be accepted as evidence of active rickets is persisting craniotabes. This disappears within a few weeks when the normal process of calcium metabolism is re-established, but enlarged epiphyses may remain for years and bent bones for a life time. A single $\mathrm{X}$-ray plate, on the other hand, will often determine whether the disease is active, healing or healed.

\section{Conclusions.}

The writer's conclusions are as follows:-For early cliagnosis in the future reliance is likely to be placed on the results of blood examination. Clinical diagnosis, based on bone changes (e.g., an enlarged rosary, and in the young infant craniotabes), can be made early, and radiographic signs are usually somewhat later than the earliest clinical signs. An enlarged rosary and large epiphyses may, however, be signs of scurvy. Unless there is craniotabes present, the stage of the disease cannot be determined by a single clinical examination, but can frequently be settled by examination of a single $\mathrm{X}$-ray plate; and radiographic diagnosis is much less subjective than the clinical. There is no doubt that the true incidence of rickets is higher than figures based on radiographic examination alone would indicate. How much higher it is impossible to say, but in the writer's opinion it is probable that perhaps twice as many cases of rickets might be detected by clinical examination as can be revealed radiographically, but this statement is based on impression and has not been tested on any large number of cases. Had it not been for the opportunity in Vienna, already mentioned, of comparing clinical and histological findings, the writer would have passed as normal many infants which, in the light of this experience, she would now class as very early rachitic cases. 